\title{
Arte y anarquismo: aproximaciones al espacio artístico en el cambio del siglo XIX al $\mathrm{XX}$
}

\section{Artículo de revisión}

\section{Paúl Marcelo Velásquez Sabogal}

Universidad Estatal de San Petersburgo, Rusia st073511@student.spbu.ru

Recibido: 11 de marzo de 2021

Aceptado: 15 de abril de 2021

Como citar este artículo: Velásquez, S. Paul Marcelo (2022). Arte y anarquismo: aproximaciones al espacio artístico en el cambio del siglo XIX al XX. Calle14: revista de investigación en el campo del arte. 17(31), pp. 66-79. DOI: https://doi.org/10.14483/21450706.18691

\section{Información}

Una versión previa de este artículo se presentó a manera de ponencia en la Conferencia Internacional de estudiantes, doctorandos y jóvenes investigadores: Lomonosov 2021, llevada a cabo por la Universidad Estatal de Moscú (12-23 de abril de 2021)»

\section{(2) (1)}

https://creativecommons.org/licenses/by/4.0/deed.es 


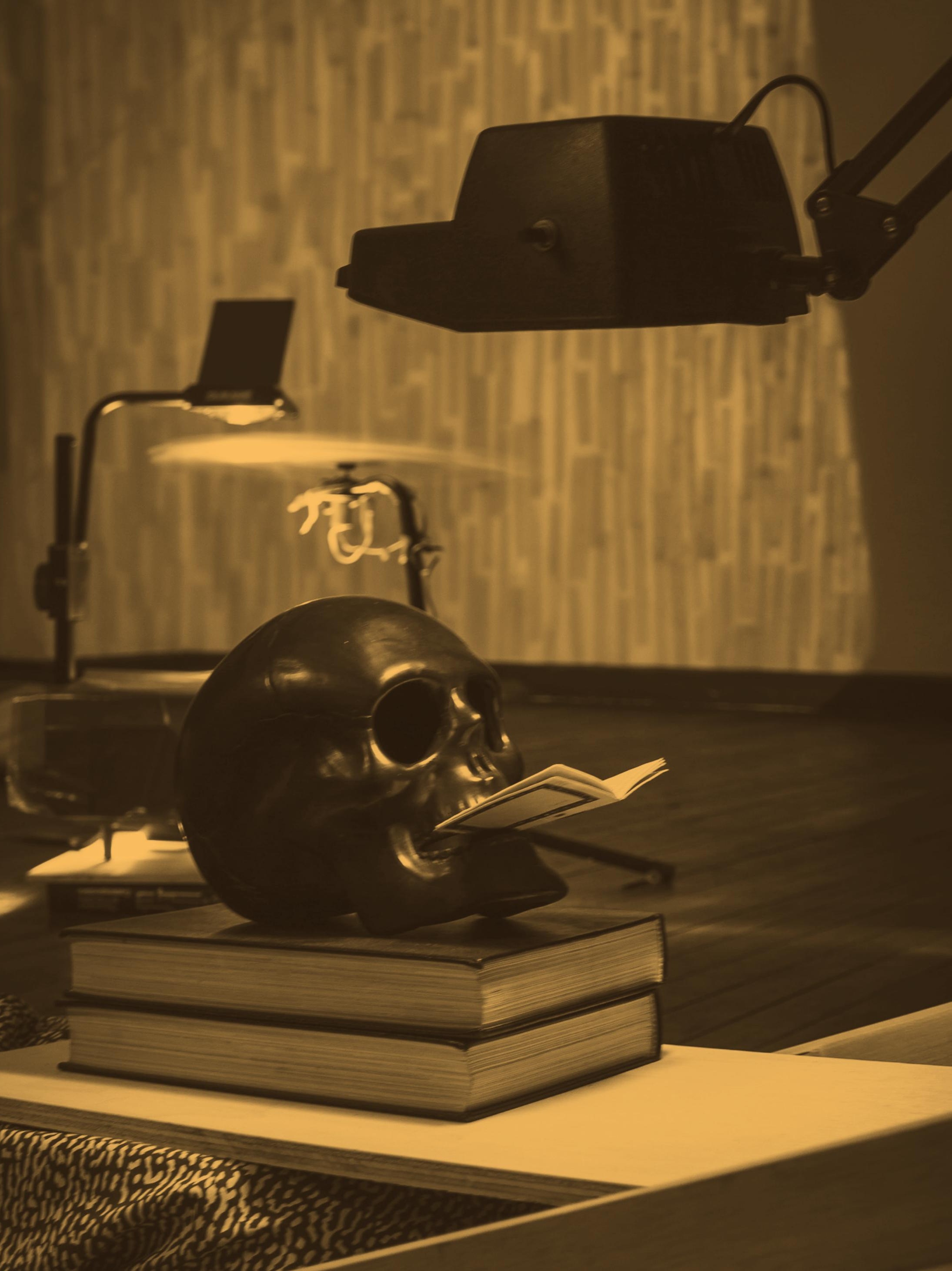




\title{
Resumen
}

La relación entre arte y anarquismo en el cambio del siglo XIX al XX despierta especial interés en los estudios contemporáneos sobre la historia del arte. En este artículo se abordan las principales tendencias de análisis que han encarado las vanguardias artísticas. Por lo tanto, se consideran las aproximaciones de diferentes autores sobre el lugar de la ideología anarquista en la formación del simbolismo, el neoimpresionismo, el cubismo, el dadaísmo, el surrealismo, el futurismo italiano, la vanguardia rusa y el expresionismo, asi como de estéticas modernistas en Argentina, México, EEUU, Rusia y Francia. La sistematización y articulación bibliográfica permite caracterizar una corriente de estudio, polémica y renovadora, sobre un periodo de la historia del arte objeto de interpretaciones canónicas y repetitivas. Como resultado se provee un aparato crítico y metodológico sobre un tema poco indagado en la literatura hispanoamericana sobre el tema.

\section{Palabras clave}

Arte y anarquismo; cambio de siglo; historia del arte anarquista; vanguardias artísticas

Art and anarchism: approaches to the artistic space at the turn of the $20^{\text {th }}$ century

\begin{abstract}
The relationship between art and anarchism at the end of the $19^{\text {th }}$ and beginning of the $20^{\text {th }}$ century arouses special interest in contemporary studies on the history of art. The article addresses the main trends of thought used to analyze the artistic avant-garde. Therefore, we will consider the approaches of different authors about the place of the anarchist ideology in the formation of Symbolism, Neo-Impressionism, Cubism, Dadaism, Surrealism, Italian Futurism, the Russian avant-garde and Expressionism, as well as of Modernist aesthetics in Argentina, Mexico, the US, Russia and France. The bibliographic systematization and articulation allow us to characterize a polemic and renovating current of study on a period of art history that is object of canonical and repetitive interpretations. As a result, a critical and methodological apparatus is provided on a subject little investigated in the Latin American literature on the subject.
\end{abstract}

\section{Keywords}

Art and anarchism; turn of the XXth century; anarchist art history; artistic vanguards

Art et anarchisme : approches de l'espace artistique au tournant du XXe siècle

\section{Résumé}

La relation entre l'art et l'anarchisme à la fin du XIXe et au début du XXe siècle suscite un intérêt particulier pour les études contemporaines sur l'histoire de l'art. L'article aborde les principaux courants de pensée qui ont essayé d'analyser l'avant-garde artistique. Ainsi, nous envisagions les approches de différents auteurs sur la place de l'idéologie anarchiste dans la formation du symbolisme, du néo-impressionnisme, du cubisme, du dadaïsme, du surréalisme, du futurisme italien, de l'avant-garde russe et de l'expressionnisme, ainsi que l'esthétique moderniste en Argentine, le Mexique, les États-Unis, la Russie et la France. La systématisation et l'articulation bibliographique permettent de caractériser un courant d'étude polémique et rénovateur sur une période de l'histoire de l'art qui fait l'objet d'interprétations canoniques et répétitives. En conséquence, un appareil critique et méthodologique est fourni sur un sujet peu étudié dans la littérature latino-américaine.

\section{Mots clés}

Art et anarchisme ; tournant du XXe siècle ; histoire de l'art anarchiste ; avant-gardes artistiques 


\section{Resumo}

A relação entre arte e anarquismo na mudança do século XIX para o XX desperta um interesse especial nos estudos contemporâneos sobre a história da arte. No artigo se abordam as principais tendências de análise à luz das vanguardas artísticas. Portanto, se consideram as aproximações de diferentes autores sobre o lugar da ideologia ácrata na formação do simbolismo, neoimpressionismo, cubismo, dadaísmo, surrealismo, futurismo italiano, da vanguarda russa e do expressionismo; assim como de estéticas modernistas na Argentina, México, EUA, Rússia e França. A sistematização e articulação bibliográfica permite caracterizar uma corrente de estudo, polêmica e renovadora, sobre um período da história da arte como objeto de interpretações canônicas e repetitivas. Como resultado fornece-se um aparato crítico e metodológico sobre um tema pouco indagado na literatura hispano-americana.

\section{Palavras chave}

Arte e anarquismo; mudança de século; história da arte anarquista; vanguardas artísticas

\section{Ruraikuna sapalla tapuchispa kaita kanchama kawachiska kai watakunapi XIX chasallata XX}

\section{Maillallachiska}

Kai iskai ruraskata kawaspa pasariska kai watakunapi XIX XX rigchachi akusinama tapuchispa kawangapa ñugpata ruraskata, kilkaska ministiduta kawangapa punchasinama ahcka ruraikunata kaipi Achka runakuna apachinkuna, paikunapa kausaita pangapi ruraspa, suma kawachispa Achka Colorkuna churaspa sugrigcha ruraikuna kanchapi kawachingapa kukunkuna kausaskapi kaskasina, chasa apachingapa, nugpasinama, Argentina, México, EE.UU, Rusia, Francia sutipi kaipi tuparinaku iachakungapa pikunami kankuna kai ruradurkuna tapuchingapa maikanmi pangapi kilkaska, hispanoamerica sutipi.

\section{Rimangapa Ministidukuna}

Rurai nukalla; trukarii ñugpamanda ruaikata sapalla katichiska; achaka ruraikuna apachig 


\section{Introducción}

El creciente interés hacia los archivos del anarquismo clásico (XIX-XX) en el ámbito científico de las últimas cuatro décadas coincide con la emergencia de nuevas tendencias historiográficas. En este escenario la historia del arte ocupa un lugar preponderante. En 1897 Max Nettlau reconoce la "potencia del movimiento libertario y revolucionario que anima la ciencia, la literatura y el arte" (p. 225). Así mismo, en la primera mitad del siglo $X X$, las obras de Herbert Read predicen la consolidación de corrientes de investigación sobre el anarquismo en los estudios contemporáneos sobre la historia del arte.

Más de medio siglo después se publica The Continuum Companion to Anarchism, que incluye las discusiones más actuales sobre el tema en la academia anglosajona. Ruth Kinna, editora y una de las autoras de la colección, plantea el contacto con la historia del arte como uno de los problemas principales. De manera acertada, también señala que este particular diálogo confronta las corrientes canónicas de análisis de la historia del arte, en cuyo seno un considerable grupo de especialistas desconoce el decisivo rol del anarquismo en el espacio artístico y cultural del cambio de siglo (Antliff et al., 2012).

En consonancia, Allan Antliff (2012), participante de esta colección, devela el lugar marginal al que "historiadores del arte de orientación marxista" (p. 73) han relegado el anarquismo, impidiendo aproximaciones disidentes. Aunque en un ensayo del autor marxista Meyer Schapiro (1935) sobre La Grande Jatte de Georges Seurat encontramos una problematización sobre la relación de esta obra con el problema de las clases sociales, así como sobre el anarquismo como una ideología común al círculo del artista, su aproximación no profundiza en este aspecto. En ensayos posteriores la ideología ácrata no figurará. En este orden, A. Antliff propone la formación de una "historia del arte anarquista", contraria a corrientes de interpretación autoritarias y a favor de una renovación metodológica (Antliff et al., 2012, p. 73). Podemos rastrear este proceso desde la segunda mitad del siglo XX hasta nuestros días.

La ausencia de un análisis historiográfico al respecto en la literatura hispanoamericana reclama la reconstrucción de las principales tendencias de estudio de un tema aún marginal en los círculos académicos. Desarrollaremos dos ejes. En el primero, se abordará el lugar del anarquismo en las vanguardias artísticas, en el ejemplo del simbolismo, el neoimpresionismo, el cubismo, el dadaísmo, el surrealismo, el futurismo italiano, la vanguardia rusa y el expresionismo. En el segundo, se discutirá la recepción de la ideología ácrata en el arte moderno de Argentina, Rusia, EEUU, México y Francia. Común a ambos momentos será la problematización de la cultura periodística anarquista como principal fuente documental tanto teórica como visual.

\section{Problemas de la estética anarquista: migraciones entre lo político y lo estético}

Uno de los primeros autores en abordar este interrogante es André Reszler (1973). En su libro L'Esthétique anarchiste se esbozan los principios teórico-filosóficos con que Pierre-Joseph Proudhon, León Tolstoi, Jean Grave, Richard Wagner, Oscar Wilde, Geoges Sorel, Mijail Bakunin, William Godwin, Piotr Kropotkin, Friedrich Nietzsche y Rudolf Rocker reflexionaron de manera directa o indirecta sobre la función social del arte y su potencial revolucionario desde una dimensión estética. Así mismo, el autor incluye las perspectivas de Karl Marx y Friedrich Engels. Estos nombres serán retomados en indagaciones de diversos académicos, creando cierto cánon teórico. ${ }^{1}$ Michael Scrivener (1979) distingue tres vertientes de la estética anarquista: la "autonomía artística", el "ideal social de un arte inalienable" y el "arte como crítica social" (p. 7). Sin temor a una reducción teórica podemos agruparlas bajo las dos tendencias con que A. Reszler enmarca el panorama del arte ácrata.

Ambas tendencias responden a una pluralidad atípica a otras estéticas socialistas. Por un lado, una tendencia "individualista" que "exalta las potencias y cualidades creativas" del individuo y su "originalidad". Por otro, una tendencia "colectivista o comunista" que aboga por "la fuerza creativa de la colectividad o el pueblo" (Reszler, 1972, p. 52). Aunque antagónicas, operan sobre la base de un "arte antiautoritario" (Reszler, 1973, p. 8). En concordancia, Lily Litvak (1981) reconoce que la estética libertaria española "exalta el individualismo, pero se siente, al mismo tiempo, portadora de la sensibilidad colectiva" (p. 10). Esta dualidad prepara el campo para el fructífero encuentro entre la vanguardia política y artística desde la primera mitad del siglo XIX. Egbert Donald (1970) rastrea este encuentro desde los utopistas socialistas, pasando por el encuentro entre P.-J. Proudhon y Gustave Courbet, hasta llegar al simbolismo,

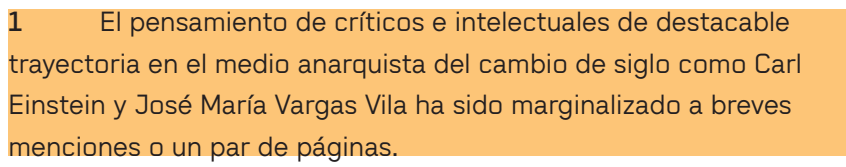


el neoimpresionismo, el cubismo, así como al futurismo temprano y la vanguardia Mexicana, en vísperas del triunfo del fascismo y el bolchevismo. Los artistas de vanguardia confluyeron con los anarquistas en los cafés, en los atentados terroristas, pero sobre todo en las publicaciones seriadas, las cuales permitieron una migración plural de valores estéticos y políticos. ${ }^{2}$

Varios autores han llevado a cabo estudios eclécticos. En este orden encontramos perspectivas panorámicas desde la Comuna de París con G. Courbet hasta la caída del muro de Berlín con Richard Mock (Antliff, 2007), sondeos colectivos sobre la estética anarquista desde las vanguardias europeas hasta grupos activistas contemporáneos de México, Dinamarca, EEUU y Argentina (McPhee y Reuland, 2007; Schleuning, 2013), así como pesquisas sobre la recepción del anarquismo en la teoría del arte (Scholz, 1999). Un poco más circunscritas son las incursiones de David Soon (1989) sobre el encuentro entre anarquistas y artistas en Montmartre, destacando las figuras de Henri Toulouse-Lautrec y O. Wilde.

En esta misma línea, David Weir (1997) proporciona una visión polémica sobre el modernismo como resultado de la migración de valores políticos del anarco-individualismo a valores estéticos. En otras palabras: "el fracaso del anarquismo aseguró el triunfo del modernismo" en tanto "varios artistas [...] le dieron una expresión estética a principios políticos" (p. 158). Una interpretación basada en la demoledora crítica de las estructuras sociales y la exaltación de un egoismo radical y nihilista de M. Stirner, que dan paso a un "individualismo estético" y una "fragmentación estilística" (pp. 169177). Aunque bajo la lupa de Matthew Arnold (2006) aquello supone la incursión del caos (la anarquía) en el orden (la cultura), esta atomización se establece como un signo común del espíritu de la modernidad. Al ser heredero del Romanticismo y del Siglo de las Luces, el artista-agoista se encuentra entre dos impulsos contrarios: el romántico M. Bakunin y el científico P. Kropotkin. El ejemplo por excelencia es Hugo Ball, sobre quien D. Weir (1997) recuerda el aspecto irracional y vitalista del dadaísmo, así como el proyecto del Cabaret Voltaire: "una institución educativa con objetivos

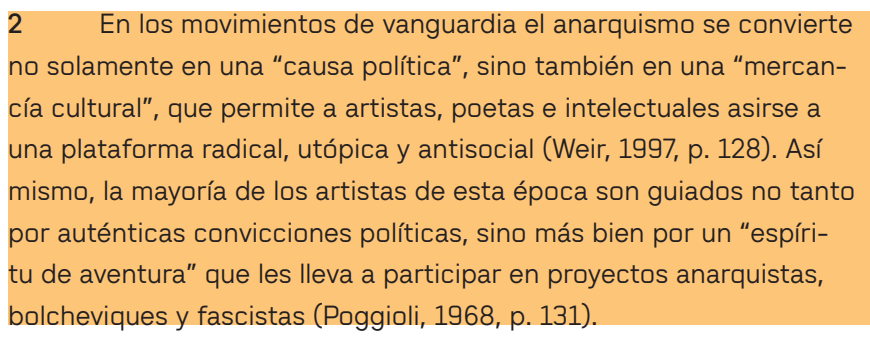

anarco-culturales" (p. 223). Estos encuentros disímiles y complementarios se dan cita en publicaciones seriadas como La Révolte, L'Endehors, Le Pére Peinard y Revue Anarchiste.

Irina Kislitsyna (2018) propone una migración inversa: de lo estético a lo político. Las perspectivas estéticas del crítico literario Vissarion Belinski operan como una influencia en la formación de las concepciones del anarquismo ruso clásico. La historiadora destaca las ideas sobre la "primacía del arte en relación a la ciencia", la "libertad de la creatividad", la "inspiración", la "espontaneidad" y el "instinto" como puentes de mutua influencia entre el crítico y M. Bakunin. Tanto la poesía popular y la cultura de la Grecia Antigua les permiten exaltar lo "primigenio" y lo "espontáneo", en cuya intersección coinciden la creatividad y la vida. Una unión azotada por las leyes de la ciencia: la teoría del arte (pp. 122-125)

Estos valores que parecen extraídos de un manifiesto dadaista nos remiten a $\mathrm{H}$. Ball y Raoul Hausmann, en cuyo ejemplo el anarquismo es mencionado como una de las cuatro corrientes políticas del dadaísmo (Van de Berg, 1999), destacando su tendencia anarco-individualista (Papanikolas, 2010). En el libro A creative passion ${ }^{3}$, Roger Farr (2010) menciona, por una parte, el activismo político del poeta $\mathrm{H}$. Ball como organizador de complejas puestas en escena musicales y teatrales de tendencia antimilitarista; y por otra parte, la asimilación del anarquismo en su pensamiento estético. En este aspecto destaca el lenguaje "como un lugar de lucha para la liberación" de estructuras sociales, pero también del lenguaje en sí mismo, más allá de su condición de “mercancía" capitalista (pp. 19-24). Así mismo, Laurent Margartin (2013) recuerda la herencia del romanticismo e idealismo alemán que subyace al impulso radical dadaísta, cuya revolución acontece en el "poema-explosión" (p. 153), pero también en el retorno a un estado primordial del hombre: la búsqueda bakuniana de un estado anterior a las estructuras sociales. En este sentido, se reconcilian tanto la "libertad política o social" como la "espiritual o interior" en una suerte de impulso religioso y libertario (Antliff et al., 2019, 99-126).

En el mismo orden de ideas, Mijail Martinov (2013) discute la interrelación del anarquismo y la vanguardia rusa temprana basado en las tensiones entre "poder"

\footnotetext{
3 Título inspirado en la frase de Jules Elysard (seudónimo de M. Bakunin): "La pasión por la destrucción es también una pasión creativa" (Bakunin, 1987, p. 226) 
y "lenguaje". Para el autor, Kazimir Malévich formula un lenguaje que en su "no-linealidad" se subleva ante "la regularidad jerárquica del mundo" (p. 168). De igual modo, es a partir de una reestructuración del lenguaje que movimientos de vanguardia rusos como el pananarquismo y el biocosmismo pretenden configurar un cambio social y estatal (2013). La discusión sobre el lenguaje como gesto antagónico contra valores sociales y estéticos ya había sido enunciada por el poeta simbolista Pierre Quillard a finales del siglo XIX tras equiparar al verso libre con la propaganda por el hecho (Reszler, 1973): un signo anarquista durante la época de los atentados.

Pablo Picasso personifica esta equivalencia. Patricia Leighten (1989) actualiza la interpretación de las influencias de movimientos de izquierda de Barcelona y Paris en la obra de pre-guerra del artista español. Si bien la migración entre política y estética sucede ya en el descubrimiento de las formas plásticas de las culturas ibérica y africana, cuyo primitivismo supone un "ataque a la complacencia burguesa, representada por la tradición clásica" (p. 81), será la revolución del lenguaje pictórico de Las señoritas de Avignon (1907), la que le valdrá el calificativo de "ataque de propaganda por el hecho", reminiscente de las "bombas de los anarquistas Catalanes" (p. 89). El cubismo mismo fue asimilado como un equivalente estético del anarquismo. El artista, por su parte, empleó recortes de periódicos sobre "temas anarquistas y de crítica social" en sus collages tempranos (p. 122). Antiestatismo, antimilitarismo y anticolonialismo son las corrientes con que usualmente se relaciona su obra, bajo la influencia de personalidades como Guillaume Apollinaire y Alfred Jarry, seguidores de la filosofía ácrata rusa.

La ya mencionada analogía de P. Quillard llama la atención sobre el anarco-simbolismo. Discutido por autores como Patrick McGuinness (2009) y Thierry Roger (2016), destaca el anarco-individualismo de M. Stirner como un puente hacia el hermetismo simbolista y la noción de arte por el arte, en tanto ruptura en el lenguaje poético. Aunque en este caso lo político no afecta lo estético, la dinamita en el lenguaje es correspondida por los ataques anarquistas, aplaudidos de manera explícita o implícita por los poetas Laurent Tailhade y Stéphane Mallarmé; ambos suscriptores y colaboradores de periódicos editados por J. Grave, P. Kropotkin y Élisée Reclus (McGuinness, 2009). Asimismo, Charles Maurin realizará una memorable xilografía del mártir anarquista Ravachol, figura alabada por el también simbolista Paul Adam en Entretiens Politiques et littéraires en 1892. Por otra parte, Bernice Rosenthal (1977) arroja luz sobre el "anarquismo místico" (p. 608), nacido del círculo simbolista de San Petersburgo a la cabeza de Georgii Chulkov y Viacheslav Ivanov como respuesta a las circunstancias de la revolución de 1905. Además del periódico ya mencionado, La Revue blanche, La Plume, Voprosy zhizni (Cuestiones de la vida), y Fakely (Antorchas), estimularon un diálogo particularmente prolífico en cuanto la formación de una mitología en torno al mártir anarquista y el periódico como bomba, así como de temas de origen mítico como la Edad dorada. Así, no es desacertado subrayar el imaginario místico y religioso como un eje transversal del anarco-simbolismo tanto en Francia como en Rusia.

En el polo opuesto nos espera la dupla del neoimpresionismo y el anarquismo. John Hutton (1994) defiende que las teorías sociocientíficas, métodos y fórmulas de creación de Paul Signac corresponden a un ideal político arraigado a las concepciones del anarco-comunismo. Años más tarde, Robyn Roslak (2007) confronta diferentes corrientes científicas de aquel entonces como el atomismo, el evolucionismo, el positivismo y el organicismo en relación con el paisaje como tema de las indagaciones sociocientíficas de este movimiento.

La dimensión ecológica de este tema es analizada por la autora a la luz de los postulados geográficos y utópicos de P. Kropotkin y É. Reclus. En este sentido, no solamente la pintura neoimpresionista es interpretada como un equivalente estético de las políticas del anarquismo, sino que la concepción misma de arte decorativo de P. Signac es asumida como un tipo de estética de orientación social. Cabe señalar que gran parte de los neoimpresionistas participaron activamente como ilustradores de periódicos anarquista como Les Temps Nouveaux, Le Sociale, entre otros ya mencionados. Sobre esta colaboración son particularmente reveladoras las aproximaciones de Anne-Marie Bouchard (2009), Aline Dardel (1987), Robert y Eugenia Herbert (1960), quienes abordan las especificidades del arte gráfico de estas publicaciones.

No menos radiante fue el anarco-futurismo en Italia. Como lo menciona Günther Berghaus (1996) resulta de suma importancia la relación del jóven Filippo Marinetti con el anarco-sindicalismo italiano, así como su amistad con los simbolistas de Montmartre, con quienes compagina desde un aspecto político antes que poético. Para Alberto Ciampi (1989) el anarco-individualismo, de inspiración nietzschiana y stirneriana, estimula un punto en común en torno la subversión del lenguaje y 
la violencia. Al respecto es especialmente reveladora la postura de Ekaterina Bobrinskaia (2015) quien identifica en la interpretación y uso de la cultura periodística como arma masiva la interrelación más clara entre el imaginario "anarquista-terrorista" y la celebración de la belleza y necesidad de la violencia del futurismo, declarada a manera de conferencia por F. Marinetti ante un auditorio ácrata en Nápoles en 1910. A pesar del desvanecimiento de esta primera influencia ideológica bajo la sombra del fascismo, podemos rastrearla en publicaciones seriadas como La Rivolta, La Sciarpa Nera, La Questione Sociale, donde Carlo Carrá participará como ilustrador (Ciampi, 1989).

A pesar de que André Breton reclama un origen anarquista para el surrealismo, este aspecto ha suscitado poco interés. Mientras que Theresa Papanikolas (2010) menciona una inspiración anarco-individualista en el surrealismo temprano, Arturo Schwarz (2014) y Pietro Ferrua (2005) arrojan luz sobre las coincidencias y tensiones a lo largo de las publicaciones de André Breton, Benjamin Péret, Jean-Louis Bédouin, Jean Schuster, entre otros, en el periódico Le Libertaire de la Federación Anarquista francesa entre 1951 y 1953. Destaca en particular el manifiesto de tono antireligioso, antimilitarista, antiestatal, anticolonial y anticapitalista: Surrealismo y anarquismo: Declaración preliminar, firmado por un numeroso grupo de artistas (entre ellos Man Ray), poetas y anarquistas, y publicado en el número del 12 de octubre de 1951 del periódico ya mencionado. Según ambos autores, el anarco-surrealismo ha permeado no solamente movimientos revolucionarios de la segunda mitad del siglo XX, sino también grupos y publicaciones de nuestros días. No obstante, representa una gran laguna, sobre la cual no se ocupan incluso en la más reciente colección de artículos sobre anarquismo y vanguardias artísticas (Antliff et al., 2019).

Sin dudas, el expresionismo ocupa un lugar de primer orden. Si bien Arthur Mitzman (1977) señala el pensamiento de Nietzsche, Freud y Stirner como un lugar de encuentro entre la cosmovisión expresionista y la anarquista, esta también tuvo lugar en una marcada tendencia medievalista. Desde la cual se redescubre el lenguaje artesanal del grabado en madera, empleado por artistas como Frans Masereel, Käthe Kollwitz, George Grosz, Otto Dix, Edvard Munch, entre otros, con un fin doble. Por un lado, la experimentación estilística y temática. Por otro, el encuentro con nuevos géneros artísticos, como la novela sin palabras en el caso del primer artista (Cohen, 1977). Ambas tendencias operan desde una preocupación de orden social, de denuncia de las atrocidades de la guerra y la modernización, así como una celebración de la libertad sexual, la vitalidad, lo femenino y la infancia (Mitzman, 1977). Este tipo de obra gráfica será publicada en periódicos anarquistas alemanes y extranjeros como Freie Jugend, Simplicissimus, Der Syndicalist, Revolution, Der Freie Arbeiter, Haro! (Bélgica) y La Protesta (Argentina), sin mencionar la confluencia de ambos ismos en Der Sturm y Die Aktion (Van den Berg, 1998).

Aunque estos movimientos se circunscriben, en gran medida, al espacio europeo, la ideología libertaria fue también un impulso desicivo en los procesos artísticos y culturales de finales del siglo XIX e inicios del XX en numerosas latitudes. Situémonos ahora en aquellos países que destacan por haber sido focos de movimientos anarquistas, cuya actividad política y activista, atravesada por una especial condición cosmopolita, permeó las estéticas modernistas de su contexto más cercano.

\section{Principales corrientes de estudio: un tema transfronterizo}

Como lo señalan Steven Hirsch y Lucien van der Walt:

El anarquismo no fue una doctrina de Europa Occidental que se difundió, perfectamente formada, hacia una 'periferia' pasiva. Por el contrario, el movimiento emergió simultánea y transnacionalmente, creado por activistas interconectados entre sí a lo largo de tres continentes: un patrón de interconección, intercambio y colaboración basado en un 'internacionalismo informal' (2010, p. liv).

De igual modo, los estudios sobre la relación entre arte y anarquismo del cambio de siglo han surgido de manera paralela en diferentes países e idiomas desde finales del siglo XX hasta nuestros días. Empero, este corpus multilingüe no siempre logra interconectarse. El enfoque nacional de numerosos investigadores tiende a obviar contextos extranjeros que pueden enriquecer la visión de lo local. Así, autores europeos y norteamericanos constantemente se citan entre sí, y los latinoamericanos miran a sus colegas más próximos. Análogamente en las conferencias suelen participar determinados círculos de académicos, evitando, quizá, cierta contaminación metodológica y temática. No obstante, la ausencia de aquel patrón de interconección refleja, más bien, la fase inicial o intermedia de una corriente de estudio de la historia del arte dispuesta a la pluralidad. Una excepción a este aislamiento es Jesse Cohn (2014), 
quien compone una cartografía cultural del imaginario anarquista desde mediados del siglo XIX hasta nuestros días en Italia, Brasil, EEUU, Argentina, Francia, Uruguay, México y España. Esta aproximación transfronteriza resulta particularmente enriquecedora como modelo metodológico y analítico. Por ahora situémonos en las incursiones del anarquismo en el arte moderno de Argentina, Rusia, EEUU, Francia y México.

Juan Suriano (2010) se detiene en la composición social de la masa trabajadora argentina como el detonante de una "estructura simbólica" basada en la "re-significación de elementos recibidos de movimientos políticos y sociales europeos" (p. 207). Lo anterior repercute no solamente en la formación de una iconografía en común basada en motivos revolucionarios, mitológicos y cristianos extranjeros, sino también en la búsqueda de un motivo local, que desemboca en la interpretación de la figura del gaucho como leitmotiv del imaginario revolucionario nacional, dando nombre incluso a la revista anarquista Martín Fierro. La tensión transfronteriza también es problematizada por María de la Rosa (2018), quien considera el pensamiento de P.-J. Proudhon, P. Kropotkin, L. Tolstoi, Jean-Marie Guyau en relación con el de anarquistas locales como Diego Abad de Santillán, Alberto Ghiraldo, Jaime Torres y Gonzalez Pacheco, como estimulante de una síntesis estética.

Silvia Dolinko (2016) devela la influencia del expresionismo alemán (Käthe Kollwitz, George Grosz y Frans Masereel) en el surgimiento y desarrollo, en la Buenos Aires de inicios de siglo, de un arte de crítica social que, basado en los medios gráficos, caldea la formación de tendencias temáticas e iconográficas. Antonio Ballester, Victor Rebuffo, Abraham Vigo, Gilllermo Hebequer, José Arato, Carlos Giambiagi, entre otros, participan activamente en la cultura periodística revolucionaria, incluyendo publicaciones anarquistas ( $\mathrm{La}$ Protesta, Acción de Arte y La Campana de palo) y de izquierda (Nervio y Contra). En la riqueza historiográfica argentina existen estudios dedicados no solamente a figuras como el crítico de arte Alfredo Chiabra Acosta (Villanueva, 2017), sino también a la dimensión cultural, artística y política de revistas como La Campana de palo (Grillo, 2008), Martín Fierro (Malosetti y Plante, 2009) e Ideas y Figuras (Rey, 2007).

Por otra parte, queda por desarrollar el problema de la migración iconográfica y la influencia estilística, temática y de géneros artísticos de artistas extranjeros a la luz de la cultura periodística anarquista en Buenos Aires. Aproximaciones en esta dirección pueden plantearse a partir de la apropiación de la obra gráfica de Jules-Félix Grandjouan en La Antorcha e Ideas y Figuras (Velásquez Sabogal, 2020), y la recepción e influencia de la obra de F. Masereel en la gráfica modernista argentina.

También México fue un territorio neurálgico para el anarquismo latinoamericano. Shelley Streeby (2013) abre el panorama de la correlación entre movimientos revolucionarios en la frontera México-EEUU en el cambio del siglo XIX al XX basada en la cultura visual en torno la Revuelta de Haymarket y el Porfiriato. El primero en el ejemplo de los esfuerzos de José Martí y Lucy Parsons por restablecer el lugar histórico de los mártires de Chicago. El segundo, a partir del libro Barbarous Mexico, cuyo material gráfico sirvió de fuente iconográfica para publicaciones como American magazine y Regeneración. La prensa revolucionaria, a su vez, permite el restablecimiento de artistas como Marius de Zayas, Ludovico Caminita y Nicolás Raveles. También Rosalía Romero (2019) aborda este problema transfronterizo en el ejemplo de la influencia anarquista en el arte moderno de ambos países, convocando figuras como el anarquista Ricardo Flórez Magón y el artista de vanguardia Doctor Alt (Gerardo Murillo). Si bien ambas aproximaciones han sido formuladas desde la lengua inglesa, destaca el estudio en español de Perla Jaimes Navarro (2012) sobre la sátira política, tanto en el aspecto visual como verbal, del periódico anarquista Regeneración.

En el caso de EEUU, A. Antliff (2001) defiende la hipótesis de que el modernismo se cimentó en la filosofía de M. Stirner, M. Bakunin y F. Nietzsche, cuyas tesis a favor de la exaltación y liberación del individuo permitieron una revuelta contra la academia. Lo anterior se materializó en "espacios expositivos alternativos, una crítica de arte politizada y la unión entre producción artística y revolución social" (p. 216), en donde destacan Ben Benn, Georges Bellows, Robert Henri, Robert Minor, Adolf Wolf, Man Ray y Rockwell Kent. Por su parte, el análisis del arte gráfico, impreso en publicaciones como Revolt, Mother earth, The Blast, The Mother School journal, TNT, Golos truda, Blast, The Egoist, The Little review, Revolutionary almanac, está supeditado a la discusión sobre pintura y escultura. Sin embargo, la gráfica cobra relevancia en la discusión sobre el proyecto editorial de Hippolyte Havel, quien incentiva la migración y apropiación de material visual proveniente de publicaciones seriadas europeas (pp. 95-122).

El mismo autor aborda la militancia de R. Minor en el anarquismo y su posterior rechazo a favor del marxismo bolchevique a finales de 1920. Asimismo, Luis González 
(2019) identifica el caso del artista español Helios Gómez, quien en 1930 abandona el anarquismo para militar en las filas comunistas, de las cuales será rechazado en 1938, regresando al frente libertario (p. 366). Para el caricaturista norteamericano esta transición significó cierto escepticismo hacia el valor revolucionario del arte. Para H. Gómez implicó un distanciamiento de los temas andaluces y gitanos, así como de la experimentación surrealista, dando lugar a un estilo más cercano al realismo socialista (González, 2019). El problema de la transición ideológica de los artistas de esta época merece un estudio historiográfico independiente. Análisis de este tipo son escasos.

Un buen ejemplo de diálogo transfronterizo en el marco de los estudios contemporáneos sobre arte y anarquismo es el número titulado "Anarchist Modernism in Print" (Antliff et al., 2013) del Journal of Modern Periodical Studies, que convoca a investigadores como Nina Gurianova, Kathy Ferguson y la ya citada P. Leighten. En este número se reflexiona a la luz del contexto francés, norteamericano y ruso tendiendo como eje las publicaciones seriadas.

En el libro The Liberation of Painting de P. Leighten (2013), se discute la interrelación entre artes visuales y anarquismo en el París de preguerra. El florecimiento de una "política de la forma" (p. 177) incentivó el desarrollo de estéticas modernistas, establecidas a partir de diferentes estrategias creativas acuñadas desde la filosofía anarquista, como: "estrategias de primitivismo y espontaneidad" (p. 7) y "estrategias de inversión" (p. 68), que robustecen la revuelta contra valores burgueses. Así, la revista anarquista L'Assiette au beurre constituyó una plataforma para la innovación tanto estilística como de géneros artísticos. En otras palabras, fue un territorio de experimentación en que la caricatura política sirvió como medio para la liberación de la pintura en el marco del cubismo (Juan Gris), el fauvismo (Kees van Dongen y Maurice de Vlaminck) y el abstraccionismo (F. Kupka). A diferencia de A. Antliff, la autora acude al arte gráfico más allá del principio propagandístico y político, lo cual hace de su trabajo un referente metodológico para investigaciones en este campo. No obstante, sus interpretaciones iconográficas deben ser consideradas con cautela, tal como lo señala la también historiadora del arte Elena Klyushina (2018) en el caso del artista K. van Dongen (p. 266).

En The Aesthetics of Anarchy, Nina Gurianova (2012) problematiza la influencia del anarquismo en la formación de la vanguardia rusa temprana, restaurándola como un movimiento independiente. En este contexto el periódico moscovita Anarkhiya (Anarquía) permite el encuentro entre el Órgano de la federación moscovita de grupos anarquistas y las discusiones sobre suprematismo, futurismo, cubismo y demás corrientes a lo largo de comunicados, anuncios, informes y manifiestos de artistas como K. Malévich, Olga Rozanova, Aleksander Rodchenko, Nadeshda Udaltsova, entre otros. Como lo advierte la autora, el modelo anarco-sindicalista fue un impulso decisivo en la concepción de asociaciones independientes y autogestionadas de artistas en aras de una relación directa con el público y del rechazo a la influencia tanto estatal como privada sobre la creatividad del artista.

De igual modo, Olga Burenina-Petrova (2016) ha llevado a cabo investigaciones fundamentales sobre la base de la revista Anarkhiya en el ejemplo de K. Malévich, A. Rodchenko y Olga Stepanova. Relucen tres aspectos. El primero, el análisis sobre el arte no-objetual, cuya obra paradigmática (Cuadraro negro de 1915) responde a una construcción de valores anarquistas. El segundo, la necesidad de un arte comunitario en A. Rodchenko y O. Stepanova reminiscente de la tendencia anarco-comunista. El tercero, la técnica del collage como un lugar de expresión antiautoritaria contra las estructuras estatales. De igual modo, la autora disecciona el "anarco-biocosmismo", un proyecto a favor de la conquista y asimilación del cosmos, la inmortalidad del hombre y el rechazo del Estado, cuyas teorías son problematizadas a través de publicaciones seriadas de la época como: Biocosmist, Bessmertiye y Anarkhiya (2019).

No obstante, el componente gráfico de otras publicaciones seriadas anarquistas rusas de inicios del siglo XX no ha despertado interés entre los historiadores del arte. Circunscribiendo el panorama a la acontecido en torno la vanguardia artística.

\section{Conclusión}

Si bien las vanguardias artísticas no pueden ser agrupadas bajo una misma tendencia ideológica, las visiones de diferentes investigadores contemporáneos restauran al anarquismo como un impulso decisivo en los procesos estéticos de una época especialmente convulsa en Rusia, Europa y el Continente Americano. Conferencias, colecciones de artículos, números temáticos de revistas y tesis de maestría o doctorado 
ratifican la consolidación paulatina de una historia del arte anarquista como corriente historiográfica polémica y renovadora.

El panorama de esta intensa migración entre lo político y lo estético ratifica las palabras de Renato Poggioli (1968) sobre el anarquismo como la "única ideología política omnipresente o recurrente en la vanguardia" ( $p$. 87). Como podemos deducir, el anarquismo constituyó un impulso decisivo en el desarrollo temprano de las vanguardias artísticas. Su rol como potencia antagónica dio paso a una revuelta contra valores sociales y estéticos de la sociedad burguesa de ese entonces, en el ejemplo no solamente de una transformación del lenguaje, sino también de la formulación de estrategias disidentes de organización entre artistas, de exposición de obra artística, así como de relación con el público.

Los manifiestos e impulsos de los artistas encontraron en el anarco-individualismo, anarco-comunismo, anarco-sindicalismo y en la prensa libertaria una correspondencia ideológica y sicológica. Si bien fue opacada por el triunfo del fascismo y el bolchevismo, hacia los cuales gran parte de los artistas de vanguardia expresaron afinidad motivados, paradójicamente, por una "mentalidad anarquista" (Poggioli, 1968, p. 99), el imaginario ácrata continuó circulando en el espíritu de la modernidad como un proyecto de radiante actualidad, cuyos postulados cobran una inusitada vigencia de cara al creciente totalitarismo de nuestros días.

En suma, este artículo es una invitación a la revisión y re-descubrimiento de un momento crucial del arte y la cultura que ha sido desgastado por interpretaciones y análisis unidireccionales y canónicos, que bajo sesgos ideológicos han marginado corrientes de desarrollo teórico, estilístico, iconográfico y de géneros artísticos. El historiador del arte contemporáneo debe insistir sobre estos destellos moribundos (no exclusivamente a la luz del anarquismo), en aras del encuentro de aquello que la historia olvidó. En nuestro caso de estudio queda por señalar al arte gráfico de la cultura periodística anarquista como uno de los grandes olvidados, cuyo análisis rara vez trasciende el principio de orientación política, eludiendo problemas más allá de lo propagandístico.

\section{Referencias}

Antliff, A. (2001). Anarchist Modernism: art, politics, and the first American avant-garde. Chicago: University of Chicago Press.
(2007). Anarchy and Art: From the Paris

Commune to the Fall of the Berlin Wall. Vancouver:

Arsenal Pulp Press.

Antliff, A., Franks, B., Gordon, U., Kinna, R., Newman

S. y Prichard, A. (2012). "Approaches to Anarchist

Research", en Ruth Kinna (ed.), The Continuum

Companion to Anarchism. London/New York: Continuum

International Publishing Group.

Antliff, M., Ferguson, K., Gifford, J. Gurianova, N., Leighten, P. y Papanikolas, Th. (2013). "Anarchist Modernism in Print", en Allan Antliff (ed.), Journal of Modern Periodical Studies, No. 2.

Antliff, M., Shryock, R., Leighten, P. y Padularosa, D. (2019). "Frictions: Aesthetics or Politics", en Caroline Kosuch (ed.), Anarchism and the Avant-Garde. Radical Arts and Politics in Perspective. Leiden: Koninklijke Brill NV.

Bakunin, M. (1987). “Reaktsiya v Germanii: Ocherk frantsuza" [Reacción en Alemania: Diario de un Francés], en B. F. Pustarnakov (comp.), Izbrannyye filosofskiye sochineniya i pis'ma [Obras filosóficas y cartas escogidas]. Moscú: Mysl' [Pensamiento].

Berghaus, G. (1996). Futurism and Politics: Between Anarchist Rebellion and Fascist Reaction, 1909-1944. Oxford: Berghahn Books.

Bobrinskaya, E. (2015). "'Krasota i neobkhodimost nasiliya': Mifopoetika rannego futurizma" ['La belleza y la necesidad de la violencia': Mitopoética del futurismo temprano], en Iskusstvoznaniye [Estudios de historia del arte], No. 1-2.

Bouchard, A.-M. (2019). "Figurer la société mourante: Culture esthétique et idéologique de la presse anarchiste illustrée en France, 1880-1914". Tesis Doctoral, Historia del Arte, Université de Montréal. Montréal. Disponible en «https://papyrus.bib.umontreal. ca/xmlui/handle/1866/4155» Consultado en diciembre de 2019.

Burenina-Petrova, O. (2016). "Anarkhiya i vlast' v iskusstve (Varvara Stepanova i Aleksandr Rodchenko)" [Anarquía y poder en el arte: Bárbara Stepanova y Aleksander Rodchenko], en Syuzhetologiya i syuzhetografiya [Sujetología y sujetografía], No. 2. 
Burenina-Petrova, O. (2019). “Bessmertiye cheloveka i telesnyye metamorfozy $v$ tvorchestve anarkhobiokosmistov" [El hombre inmortal y las metamorfosis corporales en la obra de los anarcobiocosmistas], en Quaestio Rossica, No. 1.

Carmen Grillo, M. (2008). La Campana de Palo en el campo de las revistas culturales del período vanguardista argentino (1920-1930). Buenos Aires: Academia Argentina de Letras.

Ciampi, A. (1989). Futuristi a anarchici, quali rapporti?. Pistoia: Archivio Famiglia Berneri.

Cohen, M. (1977). "The Novel in Woodcuts: A Handbook", en Journal of Modern Literature. No. 2.

Cohn, J. (2014). Underground Passages: Anarchist Resistance Culture, 1848-2011. Oakland: AK press.

Dardel, A. (1987). Les Temps nouveaux 1895-1914, un hebdomadaire anarchiste et la propagande par l'image. Paris: Éditions de la Réunion des musées nationaux.

De la Rosa, M. (2018). “La creación artística anarquista en la ciudad de Buenos Aires (1900-1930)", en Aisthesis, No. 63.

Dolinko, S. (2016). “Consideraciones sobre la tradición del grabado en la Argentina", en Nuevo Mundo Mundos Nuevos, Pictures, memories and sounds. doi:https://doi. org/10.4000/nuevomundo.69472.

Donald, E. (1970). "The idea of Avant-Garde in art and politics", en Leonardo, No. 1.

Farr, R. (2010). "Poetic License: Hugo Ball, the Anarchist Avant-garde, and Us", en Jeff Shantz (ed.), A creative passion: anarchism and culture. Cambridge: Cambridge Scholars Publishing.

Ferrua, P. (2005). “Surrealismo y Anarquismo: La colaboración de los surrealistas en Le Libertaire, periódico de la Federación Anarquista de Francia", en Plínio Coelho (comp.), Surrealismo y Anarquismo: "Proclamas surrealistas" en Le Libertaire. Buenos Aires: Libros de Anarres.

González, L. (2019). “Días de ira (1930) de Helios Gómez: La encrucijada del artista revolucionario de entreguerras", en Neophilologus, No. 103.
Gurianova, N. (2012). The Aesthetics of Anarchy: Art and Ideology in the Early Russian Avant-Garde. L.A.: University of California Press.

Herbert, R. y Herbert, E. (1960). "Artists and Anarchism: Unpublished Letters of Pissarro, Signac and Others - I", en The Burlington Magazine, No. 692.

Hirsch, S. y Van der Walt, L. (2010). “Rethinking Anarchism and Syndicalism: the colonial and postcolonial experience, 1870-1940", en Steven J. Hirsch y Lucien van der Walt (ed.), Anarchism and Syndicalism in the Colonial and Postcolonial World, 1870-1940. Leiden: Koninklijke Brill NV.

Hutton, J. (1994). Neo-Impressionism and the Search for Solid Ground: Art, Science, and Anarchism in Fin-de Siècle France. Baton Rouge: Louisiana State University Press.

Kislitsyna, I. (2018). “Esteticheskiye vzglyady V. G. Belinskogo v genezise russkogo klassicheskogo anarkhizma" [Las perspectivas estéticas de V. G. Belinski en la génesis del anarquismo ruso clásico], en Acta Eruditorum, No. 26.

Klyushina, E. (2018). "Frantsuzskaya zhurnal'naya grafika kontsa XIX - nachala XX v., etapy razvitiya, tipologiya, zhanry i stilevyye napravleniya)" [El arte gráfico de la prensa francesa de finales del siglo XIX e inicios del XX, etapas de desarrollo, tipología, géneros y corrientes estilísticas]. Tesis Doctoral, Historia del Arte, Universidad Estatal de San Petersburgo. San Petersburgo. Disponible en https://tinyurl.com/8d4xya9m Consultado en marzo de 2020.

Leighten, P. (1989). Re-Ordering the Universe: Picasso and Anarchism, 1897-1914. Princeton: Princeton University Press.

(2013). The Liberation of Painting:

Modernism and Anarchism in Avant-Guerre Paris, University of Chicago Press, Chicago.

Litvak, L. (1981). La Mirada Roja: estética y arte del anarquismo español (1880-1913). Barcelona: Antoni Bosch.

Malosetti, L. y Plante, I. (2009). "Imagen, cultura y anarquismo en Buenos Aires. Las primeras publicaciones ilustradas de Alberto Ghiraldo: de El Sol 
a Martín Fierro", en Laura Malosetti y Marcela Gené (comps.), Impresiones porteñas: imagen y palabra en la historia cultural de Buenos Aires. Buenos Aires: Edhasa.

Margantin, L. (2013). “Dada ou la boussole folle de I'anarchisme", en Dans Lignes, No. 16.

Martynov, M. (2013). "'Yazyk i vlast'. Anarkhicheskiye praktiki russkogo khudozhestvennogo avangarda" ['Lenguaje y poder'. Prácticas anarquistas de la vanguardia artística rusa], en Sbornik matitse srpske za slavistiku [Colección de estudios eslavos], No. 84.

Matthew, A. (2006). Culture and Anarchy. New York: Oxford University Press.

McGuinness, P. (2009). "Mallarmé and the Poetics of Explosion", en MLN, No. 124.

McPhee, J. y Reuland, E. (eds.). (2007). Realizing the Impossible: Art against Authority. Edinburgh: AK Press.

Mitzman, A. (1977). "Anarchism, Expressionism and Psychoanalysis", en New German Critique, No. 10.

Navarro, P. (2012). “Los usos de la sátira en la prensa anarquista mexicana del periodo revolucionario. Regeneración 1910-1918", en Pacarina del Sur, No. 11. Disponible en https://tinyurl.com/yaxve3c4 Consultado en enero de 2021.

Nettlau, M. (1897). Bibliographie de l'anarchie. Bruxelles/ Paris: Temps nouveaux/P.-V. Stock.

Papanikolas, Th. (2010). Anarchism and the Advent of Paris Dada: Art and Criticism, 1914-1924. Farnham: Ashgate.

Reszler, A. (1972). "Peter Kropotkin and His Vision of Anarchist Aesthetics", en Diogenes, No. 78.

Reszler, A. (1973). L'esthétique anarchiste. Paris: Presses universitaires de France.

Rey, A. (2007). "Apuntes para pensar el arte anarquista a través de la revista Ideas y Figuras", en Entrepasados, No. 32.

Roger, Th. (2016). "Art and anarchy in the Time of Symbolism: Mallarmé and his literary group", en Journal of the Circle for Lacanian Ideology Critique, No. 9.
Romero, R. (2019). "Anarchism and Visual Culture in Greater Mexico, 1910-1950". Tesis Doctoral, Historia del Arte, Duke University. Durham. Disponible en https://tinyurl.com/aym6jc Consultado en abril de 2020.

Rosenthal, B. (1977). "The Transmutation of the Symbolist Ethos: Mystical Anarchism and the Revolution of 1905", en Slavic Review, No. 36.

Roslak, R. (2007). Neo-Impressionism and Anarchism in Fin-de-Siècle France. Painting, Politics and Landscape. Aldershot: Ashgate.

Schapiro, M. (1935). "Seurat and 'La Grande Jatte'", en Columbia Review, No. 17.

Schleuning, N. (2013). Artpolitik: Social Anarchist Aesthetics in an Age of Fragmentation. New York: Minor Compositions.

Scholz, D. (1999). Pinsel und Dolch: Anarchistische Ideen in Kunst und Kunsttheorie, 1840-1920. Berlin: Reimer Verlag.

Schwarz, A. (2014). "Anarchia e surrealismo", en Arivista anarchica, No. 385. Disponible en «http://www.arivista. org/riviste/Arivista/385/126.htm» Consultado en enero de 2021.

Scrivener, M. (1979). "The Anarchist Aesthetic", en Black Rose, No. 1.

Sonn, D. (1989). Anarchism and Cultural Politics in Fin de Siècle France. Lincoln: University of Nebraska Press.

Streeby, Sh. (2013). Radical Sensations: World Movements, Violence, and Visual Culture. Durham/ London: Duke university press.

Suriano, J. (2010). Paradoxes of Utopia: Anarchist Culture and Politics in Buenos Aires, 1890-1910.

Oakland: AK Press.

Van den Berg, H. (1999). Avant-Garde und Anarchismus: Dada in Zürich und Berlin. Heidelberg: Universitätsverlag C. Winter.

(Agosto de 1998). Anarchism and the Opacity of German Expressionism. Propositions for a Re-evaluation of the expressionist Revolt. En Brinkhuis y Talmor [Presidencia]. V Conference of the International Society for the Study of European Ideas. Conferencia 
llevada a cabo en University for Humanist Studies, Utrecht.

Velásquez Sabogal, M. (Octubre de 2020).

Bluzhdayushcheye izobrazheniye: Zhyul'-Feliks Granzhuan i argentinskiy anarkhizm nachala XX veka" [La imagen errante: Jules-Félix Grandjouan y el anarquismo argentino de inicios del siglo XX]. En Mal'tsevoy, Stanyukovich-Denisovoy y Zakharovoy [Presidencia]. IX Mezhdunarodnoy konferentsii Aktual'nyye problemy teorii $i$ istorii iskusstva [IX Conferencia internacional: Problemas actuales de la teoría y historia del arte]. Conferencia llevada a cabo en Museo del Hermitage, Universidad Estatal de San Petersburgo y Universidad Estatal de Moscú, San Petersburgo y Moscú.

Villanueva, A. (2017). “Imagen impresa y acción social. La decoración del libro al interior del proyecto cultural de Atalaya (1922-1927)", en InMediaciones de la comunicación, No. 2.

Weir, D. (1997). Anarchy \& Culture: The Aesthetic Politics of Modernism. Massachusetts: University of Massachusetts Press. 